\title{
Escala Breve de Compaixão Santa Clara: Propriedades Psicométricas para o Contexto Brasileiro
}

\author{
Eduarda Marchetti, Caroline de Oliveira Bertolino, Cecilia Cesa Schiavon, Léia Gonçalves Gurgel, Sergio Kakuta Kato \\ Universidade Federal de Ciências da Saúde de Porto Alegre, Porto Alegre-RS, Brasil \\ Carolina Baptista Menezes \\ Universidade Federal de Santa Catarina, Florianópolis-SC, Brasil \\ Caroline Tozzi Reppold ${ }^{1}$ \\ Universidade Federal de Ciências da Saúde de Porto Alegre, Porto Alegre-RS, Brasil
}

\section{RESUMO}

O presente estudo teve como objetivo buscar evidências de validade de conteúdo e de estrutura interna e avaliar a precisão da Escala Breve de Compaixão Santa Clara para o contexto brasileiro. Os itens foram adaptados por experts e, posteriormente, foram submetidos à avaliação de juízes e amostras da população-alvo em aplicações-piloto. Os resultados dessa etapa demonstraram a equivalência semântica e logística da versão adaptada. Para avaliação de consistência interna e da validade baseada em estrutura interna, o instrumento foi preenchido por 422 indivíduos e os dados foram submetidos à análise de alfa de Cronbach e análise fatorial exploratória, respectivamente. A escala apresentou índice adequado de consistência interna $(\alpha=0,84)$ e a análise fatorial resultou em uma solução unifatorial, explicando $62,48 \%$ da variância. Os resultados psicométricos da escala adaptada foram próximos dos obtidos na escala original, indicando a pertinência do uso do instrumento em âmbito nacional.

Palavras-chave: compaixão, estudos de validação, avaliação psicológica, psicometria, comportamento pró-social.

\section{ABSTRACT - Santa Clara Brief Compassion Scale: Psychometric Properties for the Brazilian Context}

The present study aimed to find content validity and internal structure evidence, and to evaluate precision of the Santa Clara Brief Compassion Scale for the Brazilian context. The items were adapted by experts and, subsequently, were submitted for the evaluation of judges and samples with the target population in pilot applications. Results of this stage demonstrated the semantic and logistic equivalence of the adapted version. To evaluate internal consistency and validity based on internal structure, 422 individuals responded to the instrument and the data were submitted to Cronbach's Alpha Analysis and Exploratory Factor Analysis, respectively. The scale presented an adequate internal consistency index $(\alpha=0.84)$ and the factorial analysis resulted in a one-factor solution, accounting for $62.48 \%$ of the variance. The psychometric results of the adapted scale were close to those obtained by the original scale, indicating the pertinence of the use of the instrument at the national level.

Keywords: compassion; validation studies; psychological evaluation; psychometry; pro-social behavior.

\section{RESUMEN - Escala Breve de Compaixão Santa Clara: propiedades psicométricas para el contexto brasileño}

El presente estudio tiene como objetivo buscar evidencias de validez de contenido y de estructura interna y, evaluar la precisión de la Escala Breve de Compasión Santa Clara para el contexto brasileño. Para el desarrollo de la investigación los ítems fueron adaptados por expertos y posteriormente fueron sometidos a la evaluación tanto de jueces, como, por una muestra de la población objetiva en aplicaciones piloto. Los resultados de esta etapa demostraron la equivalencia semántica y logística de la versión adaptada. Para evaluación de la consistencia interna y de la validez basada en la estructura interna, el instrumento se realizó a 422 individuos y los datos fueron sometidos al Análisis de alfa de Cronbach y Análisis Factorial Exploratorio. Al final, la escala presentó un índice adecuado de consistencia interna $(\alpha=0,84)$ y el análisis factorial resultó en una solución unifactorial, explicando $62,48 \%$ de la varianza. En conclusión, los resultados psicométricos de la escala adaptada se acercan a los obtenidos en la escala original, indicando la pertinencia de uso del instrumento a nivel nacional.

Palabras clave: compasión; estudios de validez; evaluación psicológica; psicometría; comportamiento prosocial.

A compaixão é definida como uma sensibilidade individual para identificar o sofrimento nos outros, seguida por um desejo de aliviá-lo (Gilbert, 2013). Esse é um construto cuja investigação tem crescente interesse

\footnotetext{
${ }^{1}$ Endereço para correspondência: Laboratório de Pesquisa em Avaliação Psicológica. Rua Sarmento Leite, 245, Centro Histórico, 90050-170, Porto Alegre, RS Tel.: (51) 3303-8854. E-mail: carolinereppold@yahoo.com.br

Financiamento: Este estudo recebeu o apoio da Fundação de Amparo à Pesquisa do Estado do Rio Grande do Sul (FAPERGS) e do Conselho Nacional de Desenvolvimento Científico e Tecnológico (CNPq) para as bolsas de estudos dos autores.
} 
na Psicologia, sobretudo na linha dos estudos relacionados à Psicologia Positiva, cujo foco é a compreensão das forças e virtudes humanas e dos atributos que aumentam o bem-estar, levando os indivíduos a terem uma vida mais engajada, com maior propósito, realização, otimismo e emoções positivas (Bolier et al., 2013; Seligman \& Csikszentmihalyi, 2000).

Strauss et al. (2016) relatam que existem algumas etapas que caracterizam o processo de sentir compaixão, sendo elas: o reconhecimento do sofrimento do outro, o entendimento da universalidade do sofrimento, a ressonância emocional com esse sofrimento, a tolerância a sentimentos desconfortáveis e a motivação para agir de modo a aliviar esse sofrimento. Nessa perspectiva, Bornemann e Singer (2013) referem que a compaixão é um construto composto por domínios afetivos e cognitivos. Dentre os primeiros, está a capacidade de suportar ou enfrentar emoções difíceis e ser amável com as pessoas e, no segundo grupo, encontra-se a habilidade de entender o ponto de vista dos outros e de aceitar que essa perspectiva seja diferente.

Devido à proximidade conceitual, é importante diferenciar a compaixão dos construtos empatia, simpatia, altruísmo e autocompaixão. Empatia se refere à capacidade de entender o estado emocional do outro e compartilhar esses sentimentos, não necessariamente implicando uma ação (Decety \& Jackson, 2004). Simpatia se refere a uma posição de concordância com a perspectiva e com as interpretações do outro frente a seu sofrimento (Post et al., 2014). Quanto ao altruísmo, ainda existem dificuldades de compreensão em relação a essa diferença, e isso inclusive contribui para limitações na busca de estudos específicos sobre o tema. No entanto, no presente estudo, entende-se o altruísmo como uma ação que beneficia outra pessoa mas pode, ou não, estar acompanhada de, ou ser motivada por empatia ou compaixão (Batson, Ahmad, Powell, \& Stocks, 2008). Também é importante diferenciar compaixão de autocompaixão, tendo em vista a proximidade dos conceitos e observando que, no presente estudo, muito se encontrou nas bases de dados sobre autocompaixão e pouco exclusivamente sobre a compaixão, construto muitas vezes estudado mais com caráter religioso do que científico.

A autocompaixão, por sua vez, envolve o próprio sofrimento, sob a observação de três aspectos: 1. autogentileza: ser amável e compreensivo consigo mesmo; 2. humanidade compartilhada: entender a própria experiência como parte de uma experiência humana maior; e 3. mindfulness (atenção plena): habilidade de ter maior consciência e aceitação dos próprios sentimentos e pensamentos (Neff \& Germer, 2013). Já a compaixão é definida no instrumento da pesquisa em questão como comportamentos de preocupação, ternura e cuidado para com o outro, que expressem a oferta de ajuda e compreensão (Hwang, Plante, \& Lackey, 2008; Sprecher \& Fehr, 2005).
Quanto aos benefícios da compaixão, estudos internacionais demonstram que esse construto está relacionado a diferentes indicadores de saúde mental. O estudo de Rushton et al. (2009), com uma amostra de profissionais da área da saúde que participaram de uma intervenção de empatia e compaixão, demonstrou que os níveis de compaixão para si e para os outros foi aumentada em pouco mais de $80 \%$, o que demonstra que a compaixão pode ser treinada. Além disso, em um estudo controlado randomizado de treino de compaixão, os participantes relataram menores níveis de preocupação e supressão de emoções e maiores níveis de compaixão, de atenção plena - por estarem mais presentes e apresentarem maior consciência de sofrimento do outro - e de felicidade possivelmente por sentirem maior conexão e cuidado com os outros (Jazaieri et al., 2014).

Nesse sentido, também por meio de uma intervenção que visava a treinar compaixão, Klimecki, Leiberg, Ricard e Singer (2014) demonstraram que os níveis de afetos positivos, recompensa e afiliação aumentaram, enquanto que os de afetos negativos diminuíram. Além disso, o treinamento de compaixão possibilitou o fortalecimento da resiliência e a diminuição de aflições por experimentar demasiada empatia. Na mesma direção, Crocker e Canevello (2008) demonstraram haver uma relação positiva e significativa entre compaixão e apoio social e, por meio de uma amostra de mulheres em doença crônica, Chapin et al. (2014) demonstraram que intervenções com foco em compaixão podem ser eficazes em aumento da aceitação de dor e diminuição da gravidade da dor crônica e da raiva que as pessoas sentiam por sua condição, o que foi confirmado por pessoas próximas dos participantes da pesquisa.

Por sua vez, o estudo de Lim e DeSteno (2016) demonstrou que pessoas que passaram por mais adversidades no passado têm uma tendência a experimentar maior compaixão pelos demais, visto que conseguem entender a perspectiva alheia e valorizar o bem-estar de seus semelhantes. Por essa razão, intervenções que promovam a compaixão têm sido de interesse a muitas instituições de formação de profissionais da saúde, especialmente àquelas com foco em cuidados paliativos.

Contudo, embora existam pesquisas internacionais que demonstrem os efeitos positivos do desenvolvimento da compaixão sobre o bem-estar social, a literatura nacional ainda é escassa de estudos sobre o tema. Essa escassez pode ser justificada pelo fato de ser um tema atual, que teve o início de seus estudos científicos há pouco tempo, e pela falta de instrumentos válidos que permitam a investigação desse construto em âmbito nacional.

$\mathrm{Na}$ perspectiva internacional, alguns dos instrumentos mais importantes disponíveis são os seguintes: The Compassion Scale (Pommier, 2010), Compassion Subscale of the Dispositional Positive Emotion Scale (Shiota, Keltner, \& John, 2006), Compassionate Love Scale 
(Sprecher \& Fehr, 2005) e a Santa Clara Brief Compassion Scale (Hwang et al. 2008). Ressalta-se que a Santa Clara Brief Compassion Scale foi construída a partir da Compassionate Love Scale de Sprecher e Fehr (2005). No entanto, a Santa Clara Brief Compassion Scale não utiliza o termo "compassionate love", pois não é direcionada somente a aferir compaixão por pessoas próximas, mas para além disso, objetiva avaliar comportamentos de compaixão frente a desconhecidos.

Para o presente estudo foi escolhido analisar a Santa Clara Brief Compassion Scale pelo fato desta permitir uma prática aplicação e boa compreensão em contextos clínicos e de pesquisa com populações de diferentes níveis sociais e de escolaridade. Criada no contexto americano, por Hwang, Plante e Lackey (2008), a Santa Clara Brief Compassion Scale apresenta alto índice de consistência interna (alfa de Cronbach de 0,90) e bons indicadores psicométricos, sendo um instrumento curto (composto por cinco itens) e de fácil compreensão linguística. $\mathrm{O}$ instrumento foi desenvolvido em contexto religioso e científico, com o objetivo de poder ser utilizado por uma vasta gama de profissionais - como clínicos, conselheiros religiosos e pesquisadores - e para diferentes populações. Os itens escolhidos para fazer parte da escala foram os que apresentaram médias moderadas $(4,28-5,48)$, alto desvio padrão $(>1,04)$ e correlações item-a-total acima de 0,75 . Após a primeira seleção de itens, foram escolhidos os que mais se referiam com o que a escala visava a medir e quatro itens se destacaram. No entanto, como essa combinação demonstrou consistência interna inferior a 0,90 , foi entendido que a versão resumida dessa escala precisava de cinco itens.

Assim, frente à lacuna de estudos com a escala no Brasil, e observando que o construto da compaixão é importante em âmbitos individuais (promovendo saúde e bem-estar) (Rushton et al., 2009; Jazaieri et al., 2014; Chapin et al., 2014) e sociais (diminuindo a vulnerabilidade de comunidades por promover conexões e vínculos interpessoais de bondade e cuidado) (Klimecki, Leiberg, Ricard, \& Singer 2014; Crocker \& Canevello, 2008), constata-se a necessidade de utilizar a escala para avaliar a compaixão em diferentes contextos e populações a fim de, futuramente, promover intervenções no sentido de acessar esse construto mais amplamente.

Dessa forma, o presente artigo apresenta a adaptação do instrumento para o português brasileiro e o estudo de análise de precisão e busca de evidências de validade de conteúdo e de estrutura interna da referida escala para o contexto brasileiro, apresentando as hipóteses de que a escala pode ser utilizada no contexto brasileiro visto que não aparenta ser de difícil compreensão ou aplicação. Além disso, como proposto pela escala original, espera-se que a escala extraia um único fator e apresente diferenças de médias importantes em suas comparações de grupos.

\section{Método}

\section{Participantes}

Para composição da amostra para realização do estudo das propriedades psicométricas da escala adaptada, a pesquisa foi divulgada em redes sociais e em e-mails universitários institucionais. Os critérios de inclusão para participação foram ter idade igual ou superior a 18 anos e ser brasileiro. O critério de exclusão foi o não preenchimento completo do questionário sociodemográfico e/ ou da escala.

Inicialmente, 507 pessoas responderam à pesquisa. Destes, 85 foram excluídos por não responderem a todo o instrumento ou por não apresentarem as características amostrais buscadas. Assim, a amostra do estudo psicométrico foi composta por 422 participantes, sendo majoritariamente mulheres. A idade dos participantes variou de 18 a 74 anos, sendo a média de idade dos participantes 29,65 anos $(D P=11,34)$. Quanto aos resultados demográficos referentes à localização, 89,3\% dos participantes são residentes da região sul do Brasil e, em relação à escolaridade, 53,1 \% dos participantes apresentaram ensino superior incompleto, $15,2 \%$ superior completo e $31,8 \%$ nível de pós-graduação.

\section{Instrumento}

Santa Clara Brief Compassion Scale (Hwang et al.,2008) é composta por cinco afirmativas em escala Likert de sete chaves, com respostas que variam entre "nada verdadeiro para mim" (1) e "muito verdadeiro para mim" (7). No estudo americano que descreve o instrumento original, o alfa de Cronbach obtido para escala foi 0,90 e a análise fatorial resultou na extração de um único fator, obtido por meio do método de rotação varimax, que explicou $71,05 \%$ da variância. $\mathrm{Na}$ análise de dados da escala original, as mulheres obtiveram escores mais altos que os homens $(M=5,02, D P=1,19$ versus $M=4,24, D P=1,16$; $\mathrm{F}(1,223)=18,14, p<, 001)$.

\section{Procedimentos}

Após o aceite do criador da escala original para que fosse realizada a adaptação e busca de evidências de validade e precisão do instrumento para a população brasileira, o grupo de Pesquisa em Avaliação Psicológica da Universidade Federal de Ciências da Saúde de Porto Alegre (UFCSPA) realizou a adaptação da escala para versão português brasileiro. Conforme procedimento proposto por Borsa, Damásio e Bandeira (2012), o processo de investigação da validade de conteúdo contou com seis etapas, sendo elas: tradução do instrumento do idioma de origem para o idioma-alvo (com a presença de dois tradutores bilíngues independentes); síntese das versões traduzidas (observando equivalência semântica, idiomática, experiencial e conceitual); avaliação da síntese por três juízes experts (doutores em Psicologia, com experiências em pesquisas 
psicométricas e com familiaridade com estudos sobre o construto); avaliação do instrumento pelo público-alvo (estudantes de graduação e pós-graduação, graduados e pós-graduados); tradução reversa; e estudo-piloto (aplicação prévia do instrumento em uma pequena amostra-alvo).

Para a coleta de dados, foi utilizada a plataforma SurveyMonkey (www.surveymonkey.com). A pesquisa atendeu a todos preceitos éticos de pesquisa proposto pela Resolução 466/12 do Conselho Nacional de Saúde e foi aprovada pelo Comitê de Ética em Pesquisa da UFCSPA sob número 2.030.738. Inicialmente, os participantes receberam o termo de consentimento livre e esclarecido e responderam a um questionário sociodemográfico, a fim de caracterizar a amostra. Posteriormente, a Escala Breve de Compaixão Santa Clara (EBCSC) foi apresentada aos participantes na Plataforma para que fosse preenchida de forma online. Em todas as etapas, o delineamento da pesquisa foi transversal e as amostras compostas para o estudo foram de conveniência.

\section{Plano de Análise de Dados}

A busca de evidências de validade de conteúdo foi realizada por meio de especialistas que, após as etapas de tradução, síntese das versões, tradução reversa do instrumento e avaliação da escala por grupos de participantes com características amostrais, analisaram a relação dos itens com o construto que a escala se propõe a medir. A fim de verificar a validade de estrutura interna do instrumento, foi realizada análise exploratória por meio de análise fatorial dos dados com rotação varimax no programa SPSS 23. A precisão da escala foi analisada por meio da análise do alfa de Cronbach. Para investigar diferenças de média de escore em relação ao sexo e ao fato de a pessoa ter ou não alguma crença religiosa, os resultados foram comparados por meio do teste $t$ de Student, e quanto ao estado civil, foi realizada a comparação por meio de análise ANOVA.

\section{Evidências de Validade de Conteúdo por meio da Adaptação Transcultural do Instrumento}

A primeira etapa da análise de validade de conteúdo, referente à tradução do instrumento do idioma de origem para o idioma-alvo (português do Brasil), foi realizada por dois tradutores bilíngues. Após, foi realizada a comparação das versões, observando equivalência semântica, idiomática, experiencial e conceitual, para ser realizada a síntese das versões, o que se refere à segunda etapa da adaptação. Logo, a escala foi analisada por experts na área de avaliação psicológica (três doutores em Psicologia, com experiências em pesquisas psicométricas e com familiaridade com estudos sobre o construto) que realizaram julgamento sobre a pertinência técnica e teórica dos itens elaborados. Todos concordaram que os itens traduzidos eram adequados para o estudo.

$\mathrm{Na}$ quarta etapa do processo de adaptação, o instrumento adaptado foi submetido à avaliação do público-alvo (20 estudantes de graduação/pós-graduação, graduados e pós-graduados, selecionados por conveniência), para avaliação da compreensão dos itens. Todos participantes consideraram os itens compreensíveis e adequados para avaliação. Após, foi realizada a tradução reversa do instrumento. Mantida a equivalência do instrumento com a escala original, foi realizado o estudo-piloto da escala, com uma amostra de 30 participantes, selecionados por conveniência a partir da divulgação da pesquisa por meio eletrônico. Por meio das etapas desse processo, foi constatado que a escala não apresentou erros gramaticais, traduções com significados confusos ou dificuldade de inserção em um novo contexto cultural, o que demonstra que não houve discrepância na adaptação da escala que pudesse comprometer o sentido dos itens em comparação com o instrumento original, e que a escala adaptada demonstra ter evidências de validade de conteúdo.

\section{Evidências de Validade com Base na Estrutura Interna das Variáveis do Teste e Estudo de Fidedignidade}

Os resultados obtidos na análise fatorial exploratória, considerando os componentes principais, rotação varimax e critério de Kaiser de autovalor maior que 1, indicaram uma solução unifatorial, que foi corroborada pelos dados do scree plot (Figura1). O fator retido explicou 62,48 \% da variância. O índice KMO foi de 0,78, demonstrando adequação amostral para realização das análises e o teste de esfericidade de Barlett foi significativo $(p<0,001)$ $(1021,717)$. Em relação à fidedignidade, a análise do alfa de Cronbach indicou que a escala apresenta índice adequado de consistência interna $(\alpha=0,84)$. A carga fatorial obtida em cada item é apresentada na Tabela 1.

\section{Comparação de Escores da Escala em Relação a Variáveis Demográficas}

As mulheres da amostra apresentaram escores significativamente mais altos do que os homens $(M=5,41$; $D P=1,02$ versus $M=5,06 ; D P=1,16, t=2,80 p<0,005$, $d$ de Cohen: 0,32 ). As análises estatísticas demonstraram que não houve diferença significativa na média do escore da escala adaptada em relação ao estado civil $(F=1,107$; $p<0,33)$ e ao fato de os participarem autorrelatarem ter ou não uma crença religiosa $(t=-1,85 ; p<0,06)$. No entanto, as pessoas que possuíam algum tipo de crença religiosa apresentaram escores mais elevados que o outro grupo, com tendência a serem significativos $(M=5,41 ; D P=1,03$ versus $M=5,21 ; D P=1,10 ; p<0,06$; $d$ de Cohen: 0,20). 


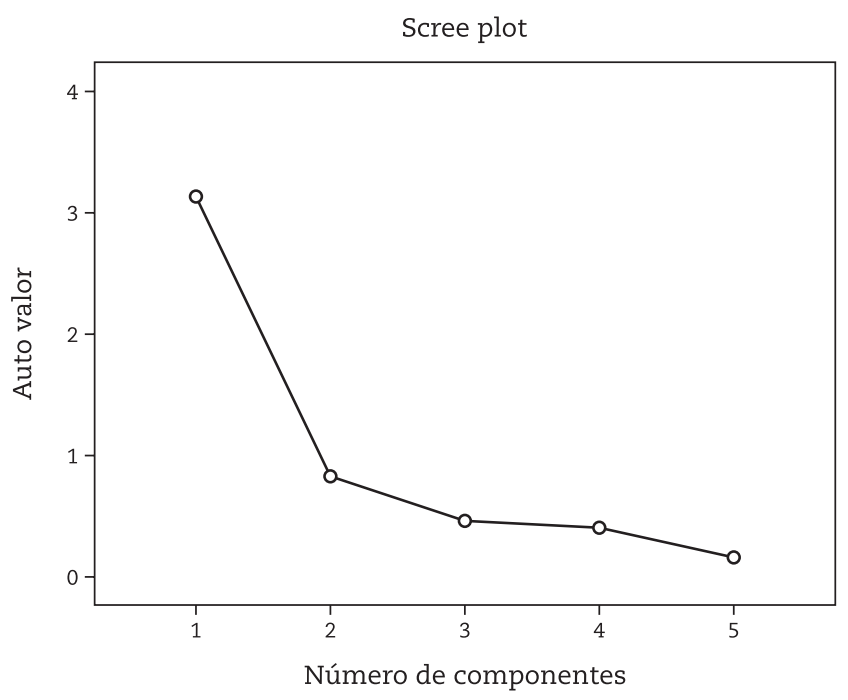

Figura 1. Scree plot da análise fatorial de EBCSC

Tabela 1

Carga Fatorial dos Itens da Escala Breve de Compaixão Santa Clara

\begin{tabular}{|c|c|}
\hline Item & Carga fatorial \\
\hline $\begin{array}{l}\text { 1. Quando eu ouço falar de alguém (um estranho) passando por } \\
\text { um momento difícil, sinto uma grande compaixão por ele/ela. }\end{array}$ & 0,71 \\
\hline 2. Tendo a sentir compaixão pelas pessoas, mesmo que eu não as conheça. & 0,76 \\
\hline $\begin{array}{l}\text { 3. Uma das atividades que proporciona maior significado à } \\
\text { minha vida é ajudar os outros quando precisam de ajuda. }\end{array}$ & 0,53 \\
\hline $\begin{array}{l}\text { 4. Prefiro me engajar em ações que ajudem outros, mesmo que sejam } \\
\text { estranhos, do que me engajar em ações que somente ajudariam a mim. }\end{array}$ & 0,44 \\
\hline $\begin{array}{l}\text { 5. Geralmente me preocupo com as pessoas (mesmo que estranhas) } \\
\text { quando estas parecem estar passando por alguma necessidade. }\end{array}$ & 0,66 \\
\hline
\end{tabular}

\section{Discussão}

Objetivo da presente pesquisa foi apresentar dados de precisão e evidências de validade de conteúdo e de estrutura interna da EBCSC para o português brasileiro. Os resultados obtidos demonstram que o estudo cumpriu seu propósito. A adaptação da escala seguiu todos os procedimentos preconizados por Borsa et al. (2012) e resultou em um instrumento equivalente ao original em termos do construto coberto pelo instrumento, da compreensão linguística e da logística da escala. $\mathrm{O}$ instrumento foi avaliado por experts e pela população-alvo, sendo considerada acessível e pertinente.

Em relação aos dados psicométricos, a versão adaptada da EBCSC manteve a estrutura unidimensional apresentada pelo estudo da escala original e permaneceu com cinco itens. A variância explicada do instrumento adaptado foi 62,48 \%, valor pouco inferior à variância explicada do único fator da escala original (71,05 \%). Da mesma forma, o estudo de precisão da versão adaptada indicou um alfa de Cronbach igual a 0,84 , considerado adequado e inferior ao instrumento original $($ alfa $=0,90)$ (Hwang et al., 2008).

Em referência à comparação de escores de compaixão por sexo, os resultados indicaram que as mulheres apresentaram maior escore na escala. Esse dado vem ao encontro de outros estudos realizados investigando esse tema. Um desses estudos foi publicado por Moore et al. (2015). Nesse artigo, os autores demonstraram que as mulheres apresentavam maiores níveis de compaixão e relatavam maior número de experiências de eventos vitais considerados negativos, as quais contribuíram para aumentar sua compaixão. Outro estudo com resultados comparáveis foi descrito por Mercadillo, Diaz, Pasaye e Barrios (2011). Na pesquisa desenvolvida por esses autores, adultos saudáveis eram experimentalmente expostos a imagens neutras e outras que evocavam compaixão. Os resultados obtidos revelaram que as 
mulheres relatam maiores níveis de compaixão e sensibilidade ao visualizarem cenas de sofrimento em relação aos homens.

O conjunto dos dados apresentados no presente artigo indicou que versão adaptada para o português brasileiro da Escala Breve de Compaixão Santa Clara é um instrumento com evidências de validade e bom índice de precisão que viabilizam seu uso para pesquisa. Trata-se de uma escala que se caracteriza como ferramenta útil, sobretudo pela sua praticidade de uso, uma vez que é composta por apenas cinco itens, os quais cobrem a definição do construto compaixão, incluindo a compaixão frente a desconhecidos, o que é um diferencial em relação a outros instrumentos disponíveis na literatura internacional que tratam da compaixão por pessoas próximas (Sprecher \& Fehr, 2005). Nesse sentido, é relevante a continuidade dos estudos com a escala adaptada, de modo que sejam obtidos dados que permitam sua utilização clínica e profissional e que possam, por exemplo, viabilizar, a partir da disponibilidade de um instrumento válido, a avaliação da efetividade de futuros programas de intervenção clínica relacionados à compaixão.

As limitações enfrentadas na realização da pesquisa foram: o fato de a amostra ser composta basicamente por mulheres, pessoas do sul do Brasil e majoritariamente estudantes saudáveis de curso superior; o caráter recente dos estudos de compaixão sob um âmbito mais científico que religioso; e a proximidade dos conceitos empatia, compaixão, simpatia, altruísmo e autocompaixão, que dificulta a busca de artigos exclusivamente sobre o tema em questão. Dessa forma, ressalta-se a importância dos estudos futuros incluírem participantes das diferentes regiões do país. Ademais sugere-se que, em futuras pesquisas, a escala seja aplicada em amostras específicas, incluindo grupos clínicos ou em contexto de vulnerabilidade social, ampliando-se assim as evidências de validade do instrumento. De qualquer forma, os dados apresentados indicam que a escala é adequada para avaliação da compaixão no contexto brasileiro e pode colaborar para o crescimento de estudos referentes ao construto em âmbito nacional, onde ainda são incipientes as pesquisas empíricas sobre o tema no campo da Psicologia ou das Ciências da Saúde, sobretudo considerando a relevância dos estudos que indicam a associação da compaixão com o desenvolvimento de comportamentos pró-sociais.

\section{Referências}

Batson, C. D., Ahmad, N., Powell, A. A., \& Stocks, E. L. (2008). Prosocial motivation. Em J. Y. Shah \& W. L. Gardner (Eds.), Handbook of motivation science (pp. 135-149). New York, NY, US: Guilford Press.

Bolier, L., Haverman, M., Westerhof, G. J., Riper, H., Smit, F., \& Bohlmeijer, E. (2013). Positive psychology interventions: A meta-analysis of randomized controlled studies. BMC Public Health, 13(1),119. doi: 10.1186/1471-2458-13-119

Bornemann, B., \& Singer, T. (2013). A cognitive neuroscience perspective: The Resource Model. Em M. Bolz, \& T. Singer (Eds.), Compassion: bridging practice and science (pp. 126-149) Munich: Max Planck Society.

Borsa, J. C., Damásio, B. F., \& Bandeira, D. R. (2012). Adaptação e validação de instrumentos psicológicos entre culturas: Algumas considerações. Paidéia, 22(53), 423-432. doi: 10.1590/S0103-863X2012000300014

Chapin, H. L., Darnall, B. D., Seppala, E. M., Doty, J. R., Hah, J. M., \& Mackey, S. C. (2014). Pilot study of a compassion meditation intervention in chronic pain. Journal of Compassionate Health Care, 1(4), 6-12. doi:10.1186/s40639-014-0004-x

Crocker, J., \& Canevello, A. (2008). Creating and undermining social in communal relationships: The role of compassionate and self-image goals. Journal of Personality and Social Psychology, 95(3), 555-575. doi: 10.1037/0022-3514.95.3.555

Decety, J., \& Jackson, P. L. (2004). The functional architecture of human empathy. Behavioral and Cognitive Neuroscience Review. 3(2):71-100. doi: $10.1177 / 1534582304267187$

Gilbert, P. (2013). The flow of life: An evolutionary model of compassion. Em M. Bolz, \& T. Singer (Eds.), Compassion: bridging practice and science (pp. 126-149) Munich: Max Planck Society.

Hwang, J. Y., Plante, T., \& Lackey, K. (2008). The development of the Santa Clara Brief Compassion Scale: An abbreviation of Sprecher and Fehr's compassionate love scale. Pastoral Psychology, 56(4), 421-428. doi: 10.1007/s11089-008-0117-2

Jazaieri, H., McGonigal, K., Jinpa, T., Doty, J. R., Gross, J. J., \& Goldin, P. R. (2014). A randomized controlled trial of compassion cultivation training: Effects on mindfulness, affect, and emotion regulation. Motivation and Emotion, 38(1), 23-35. doi: 10.1007/ s11031-013-9368-z

Klimecki, O. M., Leiberg, S., Ricard, M., \& Singer, T. (2014). Differential pattern of functional brain plasticity after compassion and empathy training. Social Cognitive and Affective Neuroscience, 9(6), 873-9. doi: 10.1093/scan/nst060

Lim, D., \& DeSteno, D. (2016). Suffering and compassion: The links among adverse life experiences, empathy, compassion, and prosocial behavior. Emotion, 16(2), 175-182. doi: 10.1037/emo0000144

Mercadillo, R. E., Diaz, J. L., Pasaye, E. H, \& Barrios, F. A. (2011). Perception of suffering and compassion experience: Brain gender disparities. Brain and cognition, 76(1), 5-14. doi: 10.1016/j.bandc.2011.03.019

Moore, R. C., Martin, A. S., Kaup, A. R., Thompson, W. K., Peters, M. E., Jestel, D. V., Golshan, S., \& Eyler, L. R. (2015). From suffering to caring: A model of differences among older adults in levels of compassion. International Journal of Geriatric Psychiatry, 30(2), 185-191. doi: $10.1002 / g p s .4123$

Neff, K., \& Germer, C. K. (2013). Self-compassion in clinical practice. Wiley Periodicals, 69(8), 856-867. doi: 10.1002/jclp.22021

Pommier, E. A. (2010). The Compassion Scale. (doctoral dissertation), University of Texas Digital Repository, Austin, USA. 
Post, S. G., Ng, L. E., Fischel, J. E., Bennett, M., Bily, L., Chandran, L., Joyce, J., Locicero, B., McGovern, K., McKeefrey, R. L., Rodriguez, J. V., \& Roess, M. W. (2014). Routine, empathic and compassionate patient care, definitions, development, obstacles, education and beneficaries. Journal of Evaluation in Clinical Practice, 20(6), 872-890. doi: 10.1111/jep.12243.

Rushton, C. H., Sellers, D. E., Heller, K. S., Spring, B., Dossey, B. M., \& Halifax, J. (2009). Impact of a contemplative end-of-life training program: Being with dying. Palliative Supportive Care, 7(4), 405-414. doi: 10.1017/S1478951509990411

Seligman, M. E., \& Csikszentmihalyi, M. (2000). Positive psychology. An introduction. American Psychologist, 55(1), 5-14. doi: 10.1037/0003066X.55.1.5

Shiota, M. N., Keltner, D., \& John, O. P. (2006). Positive emotion dispositions differentially associated with Big Five personality and attachment style. The Journal of Positive Psychology, 1(2), 61-71. doi: 10.1080/17439760500510833

Sprecher, S., \& Fehr, B. (2005). Compassionate love for close others and humanity. Journal of Social and Personal Relationships, $22(5), 629-651$. doi: $10.1177 / 0265407505056439$

Strauss, C., Taylor, B. L., Gu, J., Kuyken, W., Baer, R., Jones, F., \& Cavanagh, K. (2016). What is compassion and how can we measure it? A review of definitions and measures. Clinical Psychology Review, 47(2016), 15-27. doi: 10.1016/j.cpr.2016.05.004

\section{Sobre os autores}

Eduarda Marchetti é psicóloga, formada pela Universidade Federal de Ciências da Saúde de Porto Alegre (UFCSPA) e membro do Laboratório de Pesquisa em Avaliação Psicológica (LPAP) da UFCSPA.

Caroline de Oliveira Bertolino é psicóloga, mestre em Ciências da Saúde (UFCSPA), professora certificada pelo Santa Barbara Institute for Consciousness Studies e pela Universidade de San Diego, e membro do LPAP/ UFCSPA.

Cecilia Cesa Schiavon é nutricionista, mestre (UFSC), doutora em Ciências da Saúde (UFCSPA) e membro do LPAP/ UFCSPA.

Léia Gonçalves Gurgel é fonoaudióloga, mestre, doutora em Ciências da Saúde (UFCSPA) e membro do LPAP/ UFCSPA.

Sergio Kakuta Kato é estatístico, mestre em Epidemiologia (UFRGS), doutorando do PPG Ciências da Reabilitação (UFCSPA), professor de estatística na PUCRS e na UFCSPA membro do LPAP/ UFCSPA.

Carolina Baptista Menezes é psicóloga, mestre, doutora em Psicologia (UFRGS), professora da UFSC na graduação e na pósgraduação e coordenadora do Laboratório Psicologia Cognitiva Básica e Aplicada.

Caroline Tozzi Reppold é psicóloga, mestre, doutora em Psicologia (UFRGS), professora associada da UFCSPA na graduação e na pós-graduação, coordenadora do LPAP/UFCSPA e bolsista produtividade do CNPq. 